\title{
SIMULATION IMPLEMENTS DEMAND-DRIVEN WORKFORCE SCHEDULER FOR SERVICE INDUSTRY
}

\author{
Marcelo Zottolo \\ Edward J. Williams \\ PMC \\ 15726 Michigan Avenue \\ Dearborn, Michigan 48126, U.S.A.
}

Onur M. Ülgen

\author{
2310 Engineering Complex \\ University of Michigan - Dearborn \\ 4901 Evergreen Road \\ Dearborn, Michigan 48128, U.S.A..
}

\begin{abstract}
Simulation has long been a significant and powerful force for the improvement of manufacturing operations. More recently, it has been used to increase the efficiency, efficacy, and economy of service operations. In this case study, we describe the valuable contributions simulation made to the improvement of operations at numerous business locations of a company renting vehicles (without drivers). Specifically, discrete-event process simulation analyses played a pivotal role in the construction and implementation of the "Demand-Driven Workforce Scheduler" (DdWS) now used at the client company.
\end{abstract}

\section{INTRODUCTION}

Historically, the most frequent uses of simulation have been directed to the improvement of manufacturing operations (Law and McComas 1998). More recently, simulation has come into its own as a powerful tool for improvement of operations within the service sector of the economy (Starks and Whyte 1998). The inherent and strong abilities of simulation to analyze and assess the interaction of queuing problems well beyond the reach of closed-form analyses and formulas are of obvious value whether the queues are of parts or of people waiting for service.

In the current study, simulation was one of several analytical tools applied concurrently and synergistically to achieve improved (more accurate, more adaptable, more consistent, and more quickly obtainable) schedules of resources (typically customer-service personnel) at installations of a client company engaged in the business of renting vehicles (without drivers) to travelers. This service business is highly challenged by stiff competition, burdensome tax policies and rates, highly volatile customer demand, and high customer expectations (Horowitz and Schilling 1989). In the last few years, simulation in the service of scheduling has gradually achieved increasing visibility and success. Assessing a thorough survey of German industry at the turn of the century, (Hirschberg and Heitmann 1999) presciently remarked that simulation was achieving use for resource planning and scheduling, but lamented the lack of its use in business process analysis and improvement. Soon afterward, (Völker, Munkelt, and Gmilkowsky 2001) constructed and documented a generic approach for the construction of explicitly abridged simulation models for the purpose of mediumterm production scheduling in the manufacturing sector. As a significant example of "knowledge crossfertilization," we remark that these authors work in the discipline of business administration, not industrial engineering. More specifically, (Giribone, Mosca, and Queirolo 2003) applied simulation to the production scheduling of a medical supply company packaging line. Evolving toward the service sector, (Otamendi 2007) describes the use of simulation to improve the scheduling of geographically dispersed maintenance tasks in sprawling support networks. (Dawson et.al. 1994) discuss application of simulation modeling for staff scheduling in an Emergency Department of a Hospital.

\section{OVERVIEW OF SYSTEM AND CLIENT'S PAINS}

Customers, typically travelers, enter the client's vehiclerental system upon arrival at an airport. There, a Courtesy Bus Driver shuttles them to the vehicle-rental facility. Upon arrival, some customers may wait in a queue to see a Rental Sales Agent (RSA), obtain their rented vehicle, and leave the facility. Other rental customers may go directly to the rental lot to obtain their vehicle. At the other end of the customer service spectrum, customers returning a vehicle are acknowledged by a Greeter (GR) and shuttled back to the airport for their departing flights. Upon return, most vehicles are cleaned (interior),washed (exterior), and filled up with gasoline and windshield washing fluid by a Service Agent (SA) and returned to the rental 
lot. Some returned vehicles must undergo additional maintenance (preventive and/or corrective) before their return to the rental lot. As can well be imagined, these travelers, whether traveling for business or on holiday, are often already harried by time pressure, delayed flights, missed connections, misrouted luggage, and numerous other all-too-frequent annoyances (Levack 2007). Therefore, their service expectations are high and their tolerance for miscarriage of service very low.

In this context, the client company was experiencing multiple, chronic, and severe pains, as the consultants soon learned, when - in best doctor's bedside manner they asked the client "Where does it hurt?" These interrelated pains were over- and under-scheduling of resources, a time-consuming manual scheduling process, and overwhelmed site managers. The now happily obsolete method of scheduling personnel was a time-consuming, manual process performed in Excel $\AA$. The process was labor-intensive and inefficient and often produced an imperfect schedule. The allocation of resources was based on the manager's experience and usually the schedule produced looked the same as the previous week's schedule. Some site managers would occasionally consider in their scheduling process the corporate forecast or the volume of actual reservation for the upcoming weeks. Overall, their schedules were "flat" and ignored weekly, daily, and hourly fluctuations in the business demand. As a result, these intuition-based (or even inertia-based) schedules resulted in inflated labor costs and reduced and inconsistent service levels. Over- and under-scheduling problems were also the consequence of staffing levels misaligned with the volatile dynamics of the business demand. Visits to different client sites revealed that too many employees were assigned to departments of the facility where the demand was low for that day and those hours, and too few to areas where the demand was high. The first situation translated into excessive and unjustifiable labor costs. The second situation adversely affected the customer service level provided by having insufficient employees during the days and hours when the demand was high. Furthermore, the site manager responsible for scheduling resources was often overwhelmed, amid many other responsibilities, with the task of developing the schedules. This time-consuming responsibility would keep him or her in the back office, away from the front counter solving immediately pressing customer-related issues. Hence the scheduling task, on occasions, would itself also adversely affect the customer service level provided.

\section{CURATIVE MEASURES}

The solution was approached in steps and involved several tools from the Industrial Engineer's toolbox. The first phase of the project was standardization of the proc- esses followed by the different work groups. A fundamental lean improvement guideline states that there can be no continuous improvement without standardization (Deming 1950). Once the process was standardized and work instructions generated the process was improved by applying lean principles to the steps required to carry out a task (i.e, steps required to clean a vehicle). Using timeand-motion study methods (Aft 2001), metrics like "trips around the vehicle required to clean it" and "number of times the person cleaning a vehicle entered and exited it" were developed, animated (using the Workplace Planner ${ }^{\circledR}$ software from Proplanner; an example of this animation is shown in Figure 1), and optimized within the recommended improved process. During this project, it was of fundamental importance that the processes be standardized and optimized before schedules were constructed upon them. As the management guru Robert Townsend famously enjoined in an analogous context: "Make sure your present report system is reasonably clean and effective before you automate [computerize] it. Otherwise your new computer will just speed up the mess." (Townsend 1984). Only then was it time to build a simulation model to analyze and optimize the schedules, and ultimately to help automate the generation of them day-today and week-to-week.

\section{THE SIMULATION MODEL}

The simulation model was then built using the insights gained and lessons learned during the work assessments and process standardization. Data collection forms were developed for each of the processes and customized to reflect operational differences at each of the client's vehicle-rental facilities. Once collected, the data were fit to appropriate statistical distributions, using the software package Stat::Fit ${ }^{\circledR}$ (Hauge and Paige 2004) to properly model the variability within each process, as well as its mean duration. Enterprise Dynamics ${ }^{\circledR}$ stood out as the simulation engine of choice due to its ability to effectively and accurately model virtually any problem (Boer, de Bruin, and Verbraeck 2006), its capability of seamless connectivity to other business software, and due to its excellent three-dimensional animation capabilities, which stood the modelers of passenger railway terminals in good stead ( $\mathrm{Li}$ et al. 2006). The ability to visualize proposed solutions to the stated problems in a virtual threedimensional environment held high appeal for the client and greatly aided client acceptance. A representative example of a three-dimensional animation view is shown in Figure 2 (Appendix); this figure shows a queue of regular customers awaiting the service of an RSA to rent a vehicle. Another queue of higher-priority customers (frequent renters) is off-screen to the left. To the right in this figure, the low blue car has been returned and awaits cleaning; the green car behind it is being returned (the person 
standing beside it is a GR). Customized threedimensional icons were built to represent the airport terminals, rental office, cleaning station, vehicle wash, guard post, and the vehicles and people moving within the system. The simulation model records time stamps at key points throughout the system to track customer as well as vehicle waiting and processing times. In addition, utilization statistics are gathered for each workgroup in the system. These data were important in determining the optimal schedule for the facility staff. Specifically, schedules are generated for the four main groups of resources staffed at the car rental facility: (1) Rental Sales Agents (RSAs) who process incoming rental customers, (2) Greeters (GRs) who process customers returning their vehicles, (3) Service Agents (SAs) who clean, wash, and refuel the returning vehicles, and Courtesy Bus Drivers (CBDs) who drive customers between the rental facility and the airport. Mechanics who provide maintenance to the vehicles are also scheduled in the system but at a lower level of detail than the other resource groups. Expected rental transactions drive the schedules of RSAs. An increase in expected rentals triggers an increase in RSAs needed. Similarly, rentals and returns drive the need for GRs and SAs, and rentals and round-trip travel time drive the need for more CBDs. The output schedule depends mainly upon transaction volume and variation throughout the day and week and the service level desired at several areas during a customer's experience at the facility (for example, if the manager specifies that customers should wait no longer than four minutes to see an RSA, the software will schedule RSAs to accommodate this request).

Techniques used for the verification and validation of this model included investigatory runs of the model with all randomness removed (for easier desk-checking), stepby-step tours through model execution, examination of the animation, and structured walkthroughs (particularly of the complex operational logic which was of necessity coded within various customized objects (called atoms in ED) built for this project (and ultimately saved in a library for convenient reuse) (Sargent 2004). Most errors found and corrected during verification were within the complex code mentioned above, especially when pertinent to the exact order in which the processor performed events timetied on the current events chain (Schriber and Brunner 1998).

Subsequent to verification and validation of this model, a crucial step of the development phase lay in the user interface design of the simulation-based scheduling software, since the user experience is vital to acceptance. The main application screen, using the comfortingly familiar interface of an Excel® workbook, acts as a control panel. It clearly displays the week to schedule and facility location, and provides effective storage of records for the site via easily understood data input tabs on separate worksheets within the workbook. Users are able to accurately generate, save, retrieve, and comparatively assess multiple what-if scenario schedules quickly. During these schedule generations, the client users typically explore plausible ranges of customer demand, target resource utilizations, various cleaning and washing cycle times, service level standards (e.g., average and/or maximum time-in-queue tolerated), and the service time the customer demands with the RSA.

\section{RESULTS AND CONCLUSIONS}

The staffing schedules generated by DdWS provide the vehicle rental company with a solution to improving their scheduling system. Under- and over-staffing is evident when comparing actual past schedules with DdWS generated optimal schedules for the same periods. Resources are now staffed following daily and hourly fluctuations in business demand, resulting in a reduction in labor costs and an improvement in customer service levels across their facilities nationwide. Schedules generated (orange solid lines) for RSAs (Figure 3 in Appendix) and for GRs (Figure 4 in Appendix) explicitly illustrate the improvements now routinely achieved in schedule quality, particularly when compared to older manually generated schedules (cerise squares). The numbers of Greeters, Rental Sales Agents, and Service Agents now much more closely parallel levels of demand, whereas manual schedules chronically understaffed in the mornings and overstaffed in the afternoons. The estimated reduction in labor costs is about eight million dollars annually for the rental company in their North America facilities. Additionally, customer service levels remain high and consistent across facilities nationwide, processing times and rework have been reduced, resource utilization has risen, and site managers are much better able to adjust operations to demand levels promptly and accurately.

\section{ACKNOWLEDGMENTS}

In view of the importance of the user interface design, the authors gratefully acknowledge the contributions of Kheireddine Zeghoud, an expert in software systems development and human-computer interfaces [HCI]. The industrial engineer and colleague Justin Clark became skilled in the use of Workplace Planner ${ }^{\circledR}$, thereby contributing significantly to the engineers' and clients' understanding of work methods in use and the most promising ways to improve them. Additionally, industrial engineer and colleague Eric Lammers worked diligently on the development of the numerous interrelated portions of the Enterprise Dynamics ${ }^{\circledR}$ model itself. 


\section{REFERENCES}

Aft, Lawrence S. 2001. Measurement of Work. In Maynard's Industrial Engineering Handbook, $5^{\text {th }}$ edition, ed. Kjell B. Zandin, New York, New York: The McGraw-Hill Companies, Incorporated, 5.3-5.22.

Boer, Csaba Attila, Arie de Bruin, and Alexander Verbraeck. 2006. Distributed Simulation in Industry - a Survey Part 1 - the COTS Vendors. In Proceedings of the 2006 Winter Simulation Conference, eds. L. F. Perrone, F. P. Wieland, J. Liu, and B. G. Lawson, 1053-1060. CD ISBN 1-4244-0501-7, IEEE Catalog Number 06CH37826.

Dawson, Kathryn A., O'Conner, Kathleen, Sanchez, Pat, and Ülgen, Onur M. 1994. How to Conduct a Successful Emergency Center Staffing Simulation Study. In Proceedings of the 1994 Annual HIMSS Conference, Phoenix, Arizona, February 14-17, 1994.

Deming, William Edwards. 1950. Some Theory of Sampling. New York, New York: John Wiley \& Sons, Incorporated.

Giribone, Pietro, Roberto Mosca, and Filippo Queirolo. 2003. Validating the Production Weekly Plan by Scheduling Simulation. In Proceedings of the $15^{\text {th }}$ European Simulation Symposium, eds. Alexander Verbraeck and Vlatka Hlupic, 332-336.

Hauge, Jaret W., and Kerrie N. Paige. 2004. Learning SIMUL8: The Complete Guide, $2^{\text {nd }}$ edition. Bellingham, Washington: PlainVu Publishers.

Hirschberg, Arnd G., and Knut Heitmann. 1999. Simulation in German Industry - a Survey. In Proceedings of the $9^{\text {th }}$ European Simulation Symposium, eds. Winfried Hahn and Axel Lehmann, 429-433.

Horowitz, David, and Dana Schilling. 1989. The Business of Business. New York, New York: Harper \& Row, Publishers, Incorporated.

Law, Averill M., and Michael G. McComas. 1998. Simulation of Manufacturing Systems. In Proceedings of the 1998 Winter Simulation Conference, Volume 1, eds. D. J. Medeiros, Edward F. Watson, John S. Carson, and Mani S. Manivannan, 49-52.

Levack, Kinley. 2007. Runway Rage. In Business News 31(3):1,14 (5 March 2007)

Li, Ting, Eric van Heck, Peter Vervest, Jasper Voskuilen, Freek Hofker, and Fred Jansma. 2006. Passenger Travel Behavior Model in Railway Network Simulation. In Proceedings of the 2006 Winter Simulation Conference, eds. L. F. Perrone, F. P. Wieland, J. Liu, and B. G. Lawson, 1380-1387. CD ISBN 1-42440501-7, IEEE Catalog Number 06CH37826.

Otamendi, Javier. 2007. Simulation-Optimization in Geographic Maintenance Scheduling. In Proceedings of the $21^{\text {st }}$ European Conference on Modelling and Simulation, eds. Ivan Zelinka, Zuzana Oplatková, and Alessandra Orsoni, 62-67.
Sargent, Robert G. 2004. Validation and Verification of Simulation Models. In Proceedings of the 2004 Winter Simulation Conference, Volume 1, eds. Ricki G. Ingalls, Manuel D. Rossetti, Jeffrey S. Smith, and Brett A. Peters, 17-28.

Schriber, Thomas J., and Daniel T. Brunner. 1998. Inside Discrete-Event Simulation Software: How It Works and Why It Matters. In Proceedings of the 1998 Winter Simulation Conference, Volume 1, eds. D. J. Medeiros, Edward F. Watson, John S. Carson, and Mani S. Manivannan, 77-85.

Starks, Darrell W., and Todd C. Whyte. 1998. Tutorial: Simulation in the Hospitality Industry. In Proceedings of the 1998 Winter Simulation Conference, Volume 1, eds. D. J. Medeiros, Edward F. Watson, John S. Carson, and Mani S. Manivannan, 37-39.

Townsend, Robert. 1984. Further Up the Organization. New York, New York: Alfred A. Knopf, Incorporated.

Völker, Sven, Torsten Munkelt, and Peter Gmilkowsky. 2001. Reduced Simulation Models for MediumTerm Production Scheduling. In Proceedings of the $13^{\text {th }}$ European Simulation Symposium, eds. Norbert Giambiasi and Claudia Frydman, 187-191.

\section{AUTHOR BIOGRAPHIES}

MARCELO ZOTTOLO, born in Buenos Aires, Argentina, came to the United States to finish his college studies. He was graduated from the University of Michigan Dearborn as an Industrial and Systems Engineer in December 2000, and subsequently earned his master's degree in the same field in June 2004. He was awarded the Class Honors distinction and his Senior Design Project was nominated for the Senior Design Competition 2001. This project studied the improvement of manufacturing processes for the fabrication of automotive wire harnesses, ultimately proposing an automation tool leading to improvements in future designs. Additionally, he was coauthor of a paper on simulation in a distribution system which earned a "best paper" award at the Harbour, Maritime, and Simulation Logistics conference held in Marseille, France, in 2001. He is currently working for PMC as a Consulting Project Manager, supervising engineers in the delivery of process improvement projects and tools including simulation projects to a variety of clients. There, his responsibilities include management of building, verifying, validating, and analyzing simulation models in Enterprise Dynamics ${ }^{\circledR}$, WITNESS $\AA$, ProModel ${ }^{\circledR}$, and SIMUL8 ${ }^{\circ}$ for large corporate clients, plus supervision of and instruction in all these tasks; he also presents in-house training seminars. His email address is mzottolo@pmcorp.com 
EDWARD J. WILLIAMS holds bachelor's and master's degrees in mathematics (Michigan State University, 1967; University of Wisconsin, 1968). From 1969 to 1971, he did statistical programming and analysis of biomedical data at Walter Reed Army Hospital, Washington, D.C. He joined Ford Motor Company in 1972, where he worked until retirement in December 2001 as a computer software analyst supporting statistical and simulation software. After retirement from Ford, he joined PMC, Dearborn, Michigan, as a senior simulation and statistical analyst and trainer. Also, since 1980, he has taught evening classes at the University of Michigan, including both undergraduate and graduate simulation classes using GPSS $/ \mathrm{H}^{\mathrm{TM}}$, SLAM II ${ }^{\mathrm{TM}}$, SIMAN ${ }^{\mathrm{TM}}$, ProModel ${ }^{\circledR}$, SIMUL8 ${ }^{\circledR}$, or Arena ${ }^{\circledR}$. He is a member of the Institute of Industrial Engineers [IIE], the Society for Computer Simulation International [SCS], and the Michigan Simulation Users' Group [MSUG]. He serves on the editorial board of the International Journal of Industrial Engineering - Applications and Practice. During the last several years, he has given invited plenary addresses on simulation and statistics at conferences in Monterrey, México; İstanbul, Turkey; Genova, Italy; Rīga, Latvia; and Jyväskylä, Finland. He has served as Program Chair of the 2004, 2005, and 2006 Summer Computer Simulation Conferences, and for the 2005 IIE Simulation Conference; and will be program chair for the simulation track of the IIE 2008 annual conference. E-mail address and university web pages: <ewilliams@pmcorp.com> and $<$ http://www-personal.umd.umich.edu/ williame $>$.

ONUR M. ÜLGEN is the president and founder of PMC, a Dearborn, Michigan, based operations and industrial engineering and software services company as well as a Professor of Industrial and Manufacturing Systems Engineering at the University of Michigan-Dearborn. He received his Ph.D. degree in Industrial Engineering from Texas Tech University in 1979. His present consulting and research interests include simulation and scheduling applications, applications of lean techniques in manufacturing and service industries, supply chain optimization, and product portfolio management. He has published or presented more that 100 papers in his consulting and research areas.

Under his leadership PMC has grown to be the largest independent productivity services company in North America in the use of industrial and operations engineering tools in an integrated fashion. PMC has successfully completed more than 5000 productivity improvement projects for different size companies including General Motors, Ford, Sony Electronics, Sara Lee, Beaumont and ST. John's Hospitals. The scientific and professional societies of which he is a member include American Production and Inventory Control Society (APICS) and Institute of Industrial Engineers (IIE). He is also a founding member of the MSUG (Michigan Simulation User Group). His email addresses are ulgen@umich.edu and ulgen@pmcorp.com. 
Proceedings of the 2007 Winter Simulation Conference

S. G. Henderson, B. Biller, M.-H. Hsieh, J. Shortle, J. D. Tew, and R. R. Barton, eds.

\section{APPENDIX}

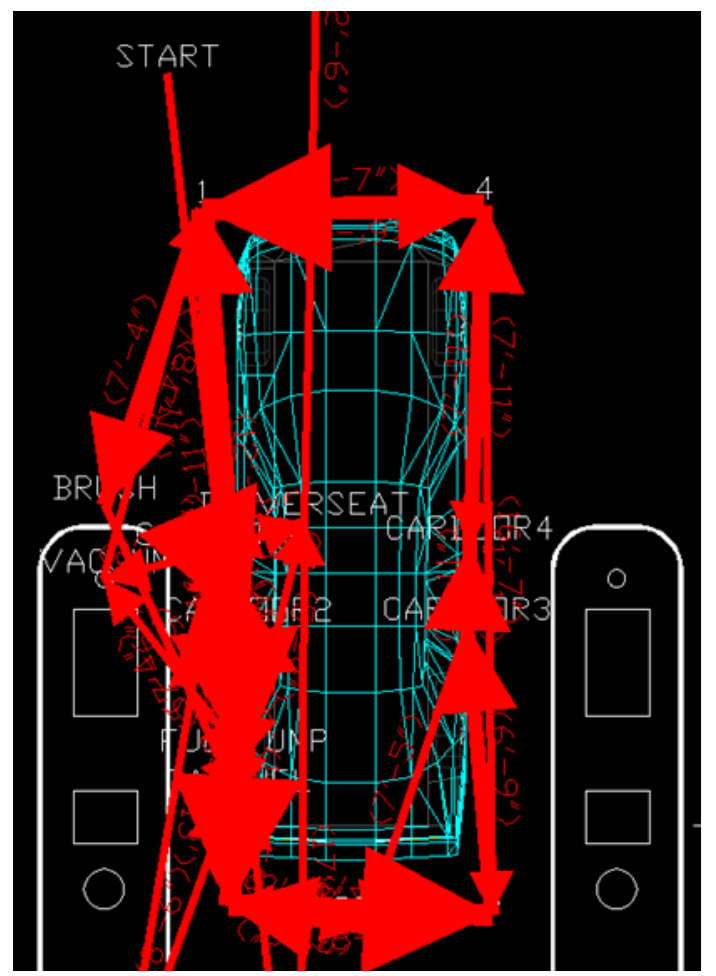

Figure 1. Workplace Planner® Animation of Interior Cleaning of Vehicle

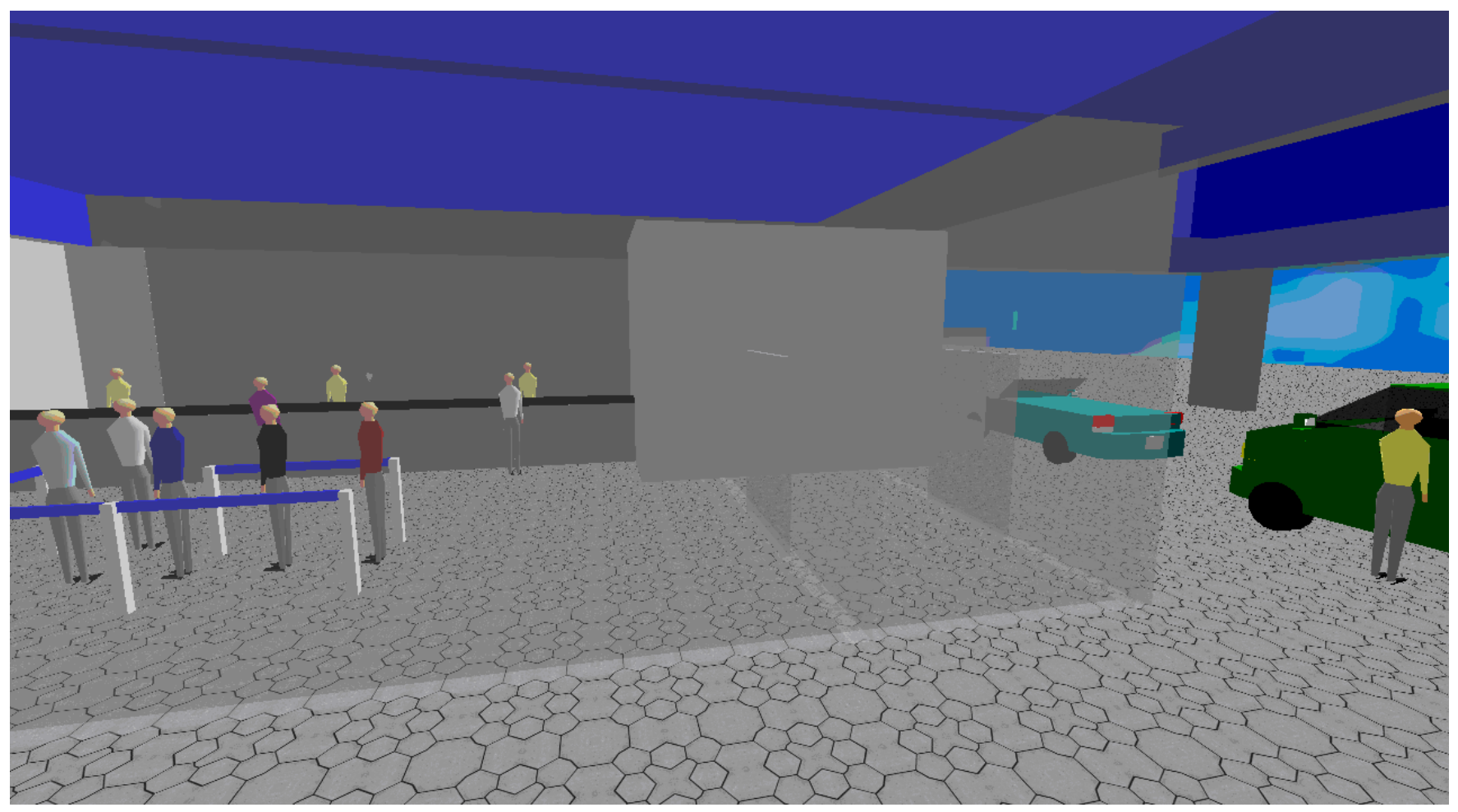

Figure 2. Animation View of Queuing Customers and Vehicles Awaiting Their Patronage 


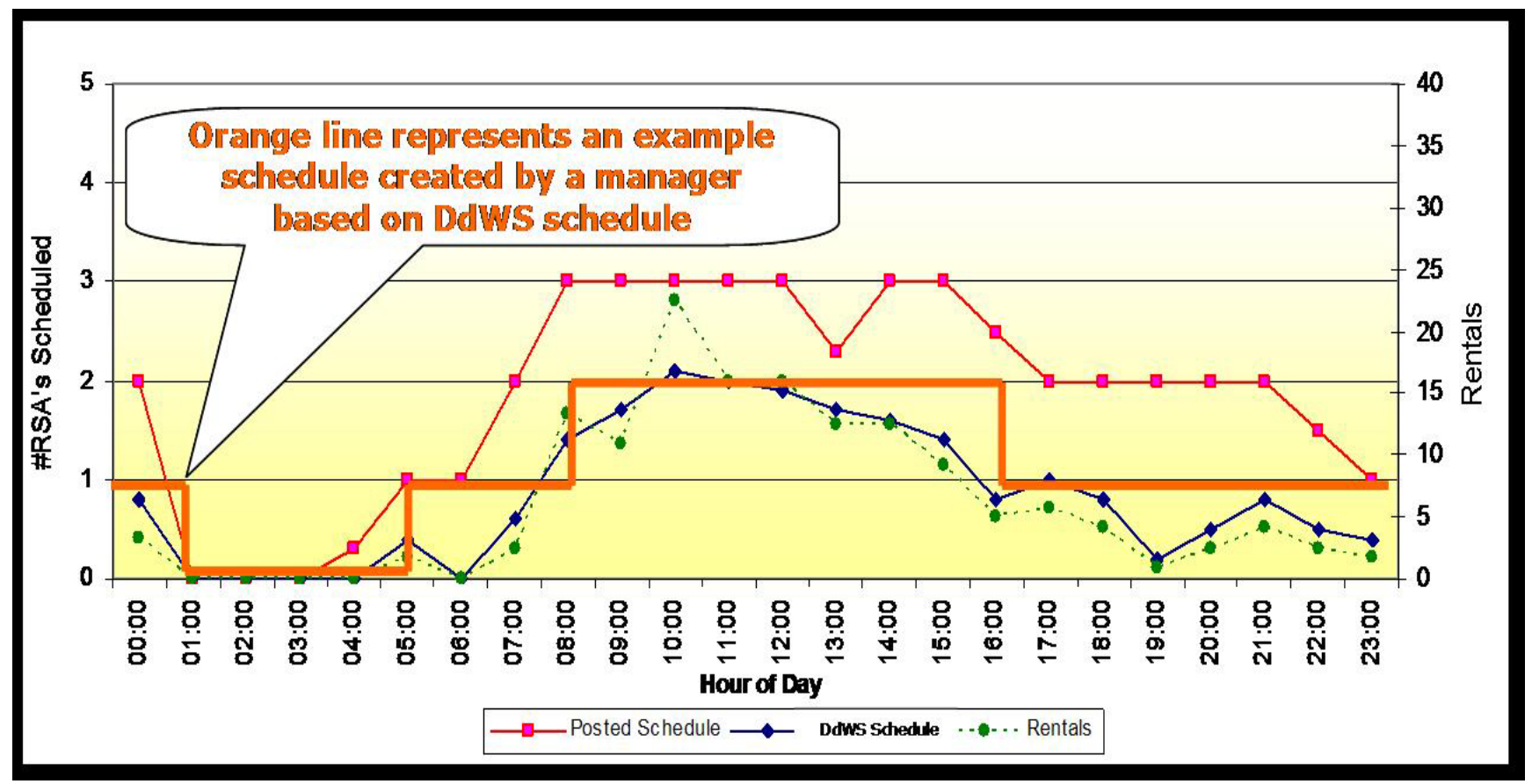

Figure 3. Schedule for Rental Sales Agents (RSAs) Specified by Demand-Driven Workforce Scheduler (DdWS)

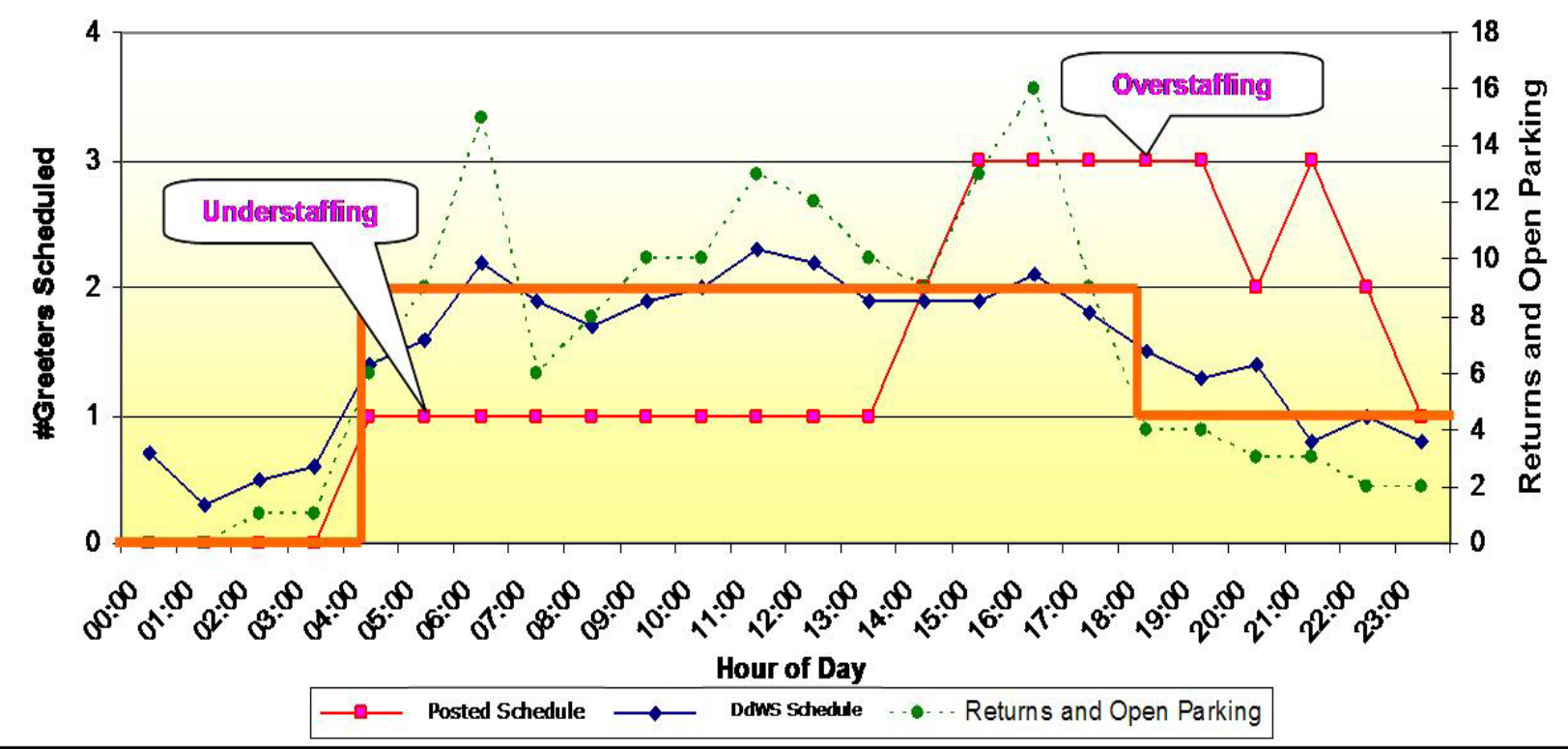

Figure 4. Schedule for Greeters (GRs) Specified by Demand-Driven Workforce Scheduler (DdWS) 